\title{
BRAID INVARIANTS AND INSTABILITY OF PERIODIC SOLUTIONS OF TIME-PERIODIC 2-DIMENSIONAL ODE'S
}

\author{
TAKASHI MATSUOKA
}

Dedicated to the memory of Juliusz P. Schauder

\begin{abstract}
We present a topological approach to the problem of the existence of unstable periodic solutions for 2-dimensional, time-periodic ordinary differential equations. This approach makes use of the braid invariant, which is one of the topological invariants for periodic solutions exploiting a concept in the low-dimensional topology. Using the braid invariant, an equivalence relation on the set of periodic solutions is defined. We prove that any equivalence class consisting of at least two solutions must contain an unstable one, except one particular equivalence class. Also, it is shown that more than half of the equivalence classes contain unstable solutions.
\end{abstract}

\section{Introduction}

Consider a 2-dimensional ordinary differential equation of the form:

$$
\frac{d x}{d t}=f(x, t)
$$

where $f: \mathbb{R}^{2} \times \mathbb{R} \rightarrow \mathbb{R}^{2}$ is a Carathéodory map (i.e., $f$ is continuous in $x$ for almost all $t$ and is measurable in $t$ for each $x$ ) which is periodic with respect to $t$ with period $\omega>0$. Assume that there exists a unique solution $x(t)$ of the initialvalue problem $x(0)=x_{0}$ for each point $x_{0} \in \mathbb{R}^{2}$ and this solution is defined on an interval containing $[0, \omega]$. We shall study the problem of the existence of unstable periodic solutions of (1). The traditional approach to this problem is to

1991 Mathematics Subject Classification. 58F22, 34C25.

Key words and phrases. Time-periodic 2-dimensional ODE, braid, unstable periodic solution.

(C)1999 Juliusz Schauder Center for Nonlinear Studies 
make the linear analysis of the related variational equation, and it is known that in some sense, the linear analysis in the instability case is easier than that in the stability case (see e.g. [1], [2]). In this paper, we present a purely topological approach to the problem. This approach makes use of the braid invariant, which is one of the topological invariants for periodic solutions exploiting a concept in the low-dimensional topology (see [4], [9] for a survey). We shall only treat periodic solutions having period $\omega$ in order to make the argument simpler.

For any solution $x(t)$ of $(1)$, the set $\{(x(t), t) \mid 0 \leq t \leq \omega\}$ becomes a simple arc in $\mathbb{R}^{2} \times[0, \omega]$ which connects a point on the plane $\mathbb{R}^{2} \times\{0\}$ to a point on the plane $\mathbb{R}^{2} \times\{\omega\}$. Thus, given a collection of finitely many $\omega$-periodic solutions, we obtain a disjoint union of simple arcs which satisfies the condition to be a braid. The braid defined in this way provides one of the topological characterizations of periodic solutions.

Assume that there are only finitely many $\omega$-periodic solutions. Then the set of all $\omega$-periodic solutions determines a braid. Using this braid, one can introduce an equivalence relation on the set of $\omega$-periodic solutions, under which two solutions are equivalent if the simple arcs corresponding to them have the same linking behavior toward any other arc. We prove that any equivalence class consisting of at least two solutions must contain an unstable one, except one particular equivalence class called the "peripheral" equivalence class. Also, when an equivalence class is not peripheral and consists of a single solution, a sufficient condition on the linking behavior of this solution is given for its instability. Furthermore, we prove that more than half of the non-peripheral equivalence classes have an unstable solution.

The content of this paper is closely related to that of a previous paper [8] of the author. The paper [8] considers an orientation-preserving embedding of the 2-dimensional closed disk into itself, and includes some results on the existence of unstable fixed points for such embeddings. Consider the case where the initialvalues of the $\omega$-periodic solutions of (1) are contained in a disk $D$ which is mapped into itself under the translation operator $U: \mathbb{R}^{2} \rightarrow \mathbb{R}^{2}$ associated with (1). Then we can apply the results in [8] mentioned above to the embedding $U: D \rightarrow D$, and we obtain several results on the existence of unstable $\omega$-periodic solutions of (1). These results are slightly stronger than those given here, since they are valid for all equivalence classes including the peripheral one. In this sense, the present paper can be regarded as a generalization of [8] to the general case where $U$ may not have an invariant disk. The proofs of the results in this paper heavily depend on results and arguments in [8], and will be given in Section 7 after some preparations on the proofs in Sections 5 and 6 .

I would like to thank Professor Jan Andres for many valuable comments. 


\section{Braids of periodic solutions}

Here we shall define a braid for a given set of $\omega$-periodic solutions. For general references on braid theory, see, e.g., [3], [6]. Let $n$ be a positive integer. We call a subset $B$ of the product $\mathbb{R}^{2} \times[0, \omega]$ an $n$-braid if the following conditions hold:

(i) $B$ is a union of mutually disjoint $n$ simple arcs.

(ii) Each arc joins a point $(x, 0) \in S \times\{0\}$ to $(\tau(x), \omega) \in S \times\{\omega\}$, where $S$ is a set of $n$ distinct points on the plane $\mathbb{R}^{2}$ and $\tau$ is a permutation defined on $S$.

(iii) Each arc intersects every plane $\mathbb{R}^{2} \times\{t\}, 0 \leq t \leq \omega$, exactly once.

These arcs are called the strings in $B$.

For an $\omega$-periodic solution $\xi$ of $(1)$, let $\operatorname{str}(\xi)$ denote the simple arc in $\mathbb{R}^{2} \times$ $[0, \omega]$ defined by

$$
\operatorname{str}(\xi)=\{(\xi(t), t) \mid 0 \leq t \leq \omega\} .
$$

We call this arc the string corresponding to $\xi$.

In this paper, we shall always assume that the equation (1) has only finitely many $\omega$-periodic solutions.

Definition 1 . Let $\mathcal{P}$ be a set of $\omega$-periodic solutions of (1), and $n$ the cardinality of $\mathcal{P}$. Since the strings corresponding to the solutions in $\mathcal{P}$ are mutually disjoint, the union $\bigcup_{\xi \in \mathcal{P}} \operatorname{str}(\xi)$ of these strings forms an $n$-braid denoted by $b(\mathcal{P})$. We call it the braid of $\mathcal{P}$.

We note that for the braid $b(\mathcal{P})$, the set $S$ in the condition (ii) is given by $S=\{\xi(0) \mid \xi \in \mathcal{P}\}$ and $\tau$ is the identity permutation.

\section{An equivalence relation on periodic solutions}

In this section, we shall introduce an equivalence relation on the set of $\omega$ periodic solutions. We first need the notion of a "block" in a braid:

Definition 2. Let $B$ be a braid. A union $B_{0}$ of strings in $B$ is called a block in $B$ if there is a subset $T$ of $\mathbb{R}^{2} \times[0, \omega]$ such that

(i) $T$ is the image of some embedding $\Lambda: D \times[0, \omega] \rightarrow \mathbb{R}^{2} \times[0, \omega]$, where $D$ is a closed disk, with $\Lambda(D \times\{t\}) \subset \mathbb{R}^{2} \times\{t\}$ for each $t$.

(ii) If we denote by $T_{t}$ the $t$-slice of $T$, i.e. the set $\left\{x \in \mathbb{R}^{2} \mid(x, t) \in T\right\}$, then we have $T_{0}=T_{\omega}$.

(iii) $B_{0}=B \cap T$.

We call $T$ an isolating tube for $B_{0}$ with respect to $B$.

ExAmple 1. It is clear that $B$ is a block in itself, and any string in $B$ is also a block in $B$. We give non-trivial examples in Figures 1 and 2 . Let $B$ be the braid consisting of three strings $s_{1}, s_{2}, s_{3}$ as in Figure 1. Then the union 
$B_{0}=s_{1} \cup s_{2}$ is a block in $B$, and the set $T$ drawn here gives an isolating tube for $B_{0}$. On the other hand, $s_{1} \cup s_{3}$ is not a block, Indeed, if it were a block, then the string $s_{2}$ winds around $s_{1}$ and $s_{3}$ in the same number of times. However, these winding numbers are 1 and 0 , respectively, and hence we get a contradiction. Consider next the braid $B$ as in Figure 2. Then $s_{1} \cup s_{2}$ and $s_{4} \cup s_{5}$ are blocks in $B$ with isolating tubes $T, T^{\prime}$, respectively. Also, $s_{3} \cup s_{4} \cup s_{5}$ is clearly a block. Furthermore, we can find an isolating block for the union $s_{2} \cup s_{3} \cup s_{4} \cup s_{5}$, and so this union is a block.

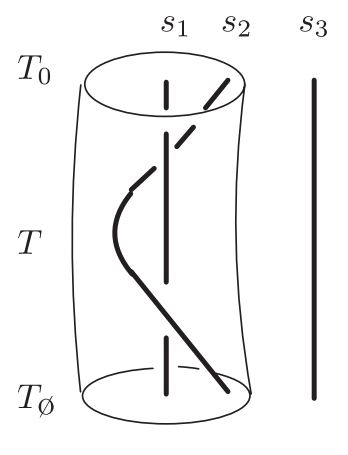

FiguRE 1

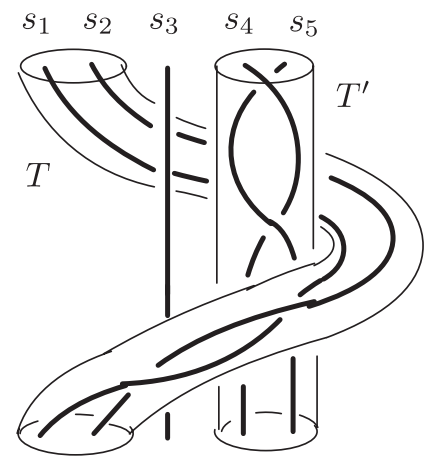

Figure 2

Let $\mathcal{P}_{\omega}$ denote the set of $\omega$-periodic solutions.

Definition 3. Two $\omega$-periodic solutions $\xi_{1}$ and $\xi_{2}$ are said to be equivalent if the braid $b\left(\left\{\xi_{1}, \xi_{2}\right\}\right)=\operatorname{str}\left(\xi_{1}\right) \cup \operatorname{str}\left(\xi_{2}\right)$ forms a block in $b\left(\mathcal{P}_{\omega}\right)$.

The choice of the term "equivalent" in this definition is reasonable as the following proposition shows:

Proposition 1. The relation on $\mathcal{P}_{\omega}$ defined above is an equivalence relation.

ExAmple 2. (a) Suppose the equation (1) has three $\omega$-periodic solutions $\xi_{i}$, $i=1,2,3$ and the braid $b\left(\mathcal{P}_{\omega}\right)$ is as in Figure 1 , where $s_{i}=\operatorname{str}\left(\xi_{i}\right)$. Then $\xi_{1}$ and $\xi_{2}$ are equivalent, since $b\left(\left\{\xi_{1}, \xi_{2}\right\}\right)=s_{1} \cup s_{2}$ is a block in $B=b\left(\mathcal{P}_{\omega}\right)$. However, $\xi_{3}$ is not equivalent to $\xi_{1}$, since $s_{1} \cup s_{3}$ is not a block. Thus, there are two equivalence classes $\left\{\xi_{1}, \xi_{2}\right\},\left\{\xi_{3}\right\}$.

(b) Secondly, suppose (1) has five $\omega$-periodic solutions $\xi_{i}, i=1, \ldots, 5$, with the braid $b\left(\mathcal{P}_{\omega}\right)=s_{1} \cup \ldots \cup s_{5}$ as in Figure 2, where $s_{i}=\operatorname{str}\left(\xi_{i}\right)$. Then, considering winding numbers also in this case, we see easily that there are three equivalence classes $\left\{\xi_{1}, \xi_{2}\right\},\left\{\xi_{3}\right\}$, and $\left\{\xi_{4}, \xi_{5}\right\}$.

It should be noted that there is one exceptional equivalence class for which our main results, which will be stated in the next section, are not valid. This is the equivalence class consisting of the "peripheral" solutions defined below: 
Definition 4. An $\omega$-periodic solution $\xi$ is said to be peripheral if one of the following conditions holds:

1. $\mathcal{P}_{\omega}=\{\xi\}$, i.e., there are no other $\omega$-periodic solutions.

2. There are at least two $\omega$-periodic solutions and $b\left(\mathcal{P}_{\omega}-\{\xi\}\right)$ is a block in $b\left(\mathcal{P}_{\omega}\right)$.

Proposition 2. The set of peripheral solutions forms an equivalence class.

We call this class consisting of all the peripheral solutions the peripheral equivalence class, and any other equivalence class a non-peripheral equivalence class. The equation (1) may not have any peripheral solution. In this case, the peripheral equivalence class is an empty set.

ExAmPle 3. If $\mathcal{P}_{\omega}$ is as in Example 2(a), then $\xi_{3}$ is peripheral, since $b\left(\mathcal{P}_{\omega}-\right.$ $\left.\left\{\xi_{3}\right\}\right)=s_{1} \cup s_{2}$ is a block. Therefore, $\left\{\xi_{3}\right\}$ is the peripheral equivalence class. Also, if $\mathcal{P}_{\omega}$ is as in Example 2(b), then $\left\{\xi_{1}, \xi_{2}\right\}$ is the peripheral equivalence class, since $b\left(\mathcal{P}_{\omega}-\left\{\xi_{1}\right\}\right)=s_{2} \cup s_{3} \cup s_{4} \cup s_{5}$ is a block and this means that $\xi_{1}$ is peripheral.

Propositions 1 and 2 will be proved in Section 7.

\section{Existence of unstable fixed points}

In this section, we shall state our main results, which are concerned with the existence of unstable $\omega$-periodic solutions. First, we recall the definition of a stable solution (cf. [7]).

Definition 5. A solution $x_{0}$ of (1) defined for $0 \leq t<\infty$ is stable (or Ljapunov stable) if for any $\varepsilon>0$, there is a $\delta>0$ such that every solution $x(t)$ with $\left|x(0)-x_{0}(0)\right|<\delta$ is defined for all $0 \leq t<\infty$ and satisfies $\left|x(t)-x_{0}(t)\right|<\varepsilon$ for any $t$. Otherwise, $x_{0}$ is said to be unstable.

TheOREm 1. Any non-peripheral equivalence class consisting of at least two $\omega$-periodic solutions contains an unstable one.

In the case of an equivalence class with only one element, the following proposition provides a sufficient condition for its instability:

Proposition 3. Suppose an $\omega$-periodic solution $\xi_{0}$ is not peripheral and is a unique element in its equivalence class. Assume that there is a subset $\mathcal{P}$ of $\mathcal{P}_{\omega}$ containing $\xi_{0}$ such that $b(\mathcal{P})$ and $b\left(\mathcal{P}-\left\{\xi_{0}\right\}\right)$ are blocks in $b\left(\mathcal{P}_{\omega}\right)$. Then $\xi_{0}$ is unstable.

Theorem 1 and Poroposition 3 would suggest that not a few equivalence classes have an unstable solution. In fact, the following theorem holds: 
THEOREM 2. More than half of the non-peripheral equivalence classes contain an unstable $\omega$-periodic solution.

Example 4. (a) Suppose $\mathcal{P}_{\omega}$ has the braid as in Figure 3. Then $\left\{\xi_{4}\right\}$ is the peripheral equivalence class, and the non-peripheral equivalence classes are $E_{1}=\left\{\xi_{1}, \xi_{2}\right\}$ and $E_{2}=\left\{\xi_{3}\right\}$. Since $E_{1}$ has two solutions, by Theorem 1 , at least one of these solutions is unstable. Also, $\xi_{3}$ satisfies the assumption of Proposition 3 with $\mathcal{P}=\left\{\xi_{1}, \xi_{2}, \xi_{3}\right\}$. Hence $\xi_{3}$ is unstable. Thus, both $E_{1}$ and $E_{2}$ contain an unstable solution.

(b) We show that the estimate of the number of equivalence classes with unstable solutions given in Theorem 2 is the best possible one, by constructing an example. Consider the self-homeomorphism $H^{\prime}$, on the disk with three disjoint open disks removed, which can be defined in a similar way to $H_{P}^{\prime}$ in $[4$, Section 1.8] by replacing the matrix

$$
\left(\begin{array}{ll}
2 & 1 \\
1 & 1
\end{array}\right)
$$

in the definition of $H_{P}^{\prime}$ by

$$
\left(\begin{array}{ll}
5 & 2 \\
2 & 1
\end{array}\right) .
$$

We get a self-homeomorphism $g$ of $\mathbb{R}^{2}$ by extending $H^{\prime}$ to the whole disk $D$ and identifying $\mathbb{R}^{2}$ with the interior Int $D$. Then, choosing an isotopy from id to $g$, we get a vector field on $\mathbb{R}^{2} \times[0, \omega]$ which induces a time-periodic equation (1). We can choose the extension of $H^{\prime}$ appropriately so that the equation (1) has five $\omega$-periodic solutions $\xi_{i}, i=1, \ldots, 5$, with the braid $b\left(\mathcal{P}_{\omega}\right)$ as in Figure 4 and $\xi_{1}, \xi_{5}$ are stable and the other three are unstable. It is easy to see that each of the five $\omega$-solutions is non-peripheral and is the unique element in its equivalence class. Therefore, there are five non-peripheral equivalence classes and exactly three of them consist of unstable solutions.

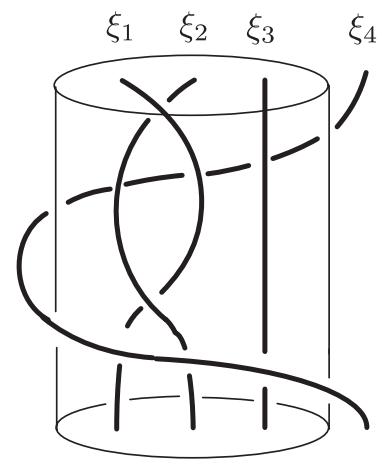

FiguRE 3

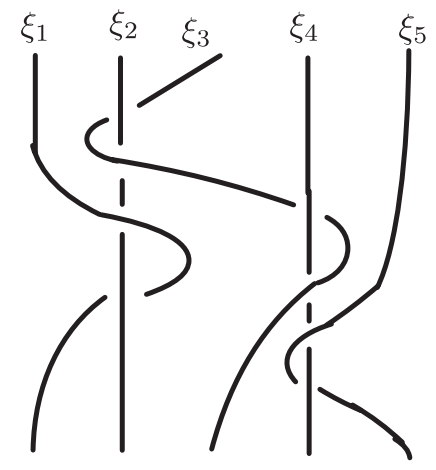

FIGURE 4 


\section{Canonical homeomorphism on a surface}

Here we explain some notions in the Nielsen-Thurston classification theory of surface homeomorphisms (see, e.g, [10], [5], [4, Section 7], which are necessary for the proofs of the results of this paper. Let $M$ be a compact surface, and $S$ a finite subset in the interior Int $M$ of $M$. A homeomorphism $\phi: M \rightarrow M$ with $\phi(S)=S$ is said to be reducible relative to $S$ if there exists a finite union $\Sigma$ of disjoint simple closed curves (called the reducing curves) in Int $M-S$ such that $\phi$ maps $\Sigma$ to $\Sigma$ and each connected component of $M-(S \cup \Sigma)$ has negative Euler characteristic. The Nielsen-Thurston classification theory states that every homeomorphism $g: M \rightarrow M$ with $g(S)=S$ is isotopic, relative to $S$, to a homeomorphism $\phi: M \rightarrow M$ with $\phi(S)=S$ which is of finite order, pseudo-Anosov, or reducible. Moreover, in the reducible case, $\phi$ is decomposed into finite-order and pseudo-Anosov components. More precisely, such $\phi$ can be chosen to have a disjoint union $\mathcal{A}$ of invariant annuli (called the reducing annuli), one around each reducing curve, such that on each connected component $N$ of $M-\mathcal{A}, \phi$ is either of finite order or pseudo-Anosov. This homeomorphism $\phi$ is called a canonical homeomorphism on $M$ relative to $S$. A connected component $N$ of $M-\mathcal{A}$ is called a component of $\phi$, and it is called a finite-order component or a pseudo-Anosov component if the restriction of $\phi$ to $N$ is finite order or pseudo-Anosov respectively.

A simple closed curve in $M$ invariant under $\phi$ is called a $\phi$-invariant curve. We can assume, without loss of generality, that $\phi$ is twisted on every reducing annulus $A$ in the sense that if $\phi$ is equal to the identity on $\partial A$, then $\phi: A \rightarrow A$ is not isotopic to id through an isotopy fixing $\partial A$ pointwise. In fact, if this does not hold for a reducing annulus $A$, then we can glue the boundaries of $A$ together eliminating $A$. Under this assumption, the following fact on $\phi$-invariant curves holds: Suppose that $\phi$ is a canonical homeomorphism which is isotopic to a homeomorphism $g$ relative to $S$. Then, for any simple closed curve $C$ in $M-S$ which is homotopic to its image $g(C)$ in $M-S$, there exists a $\phi$-invariant curve $\Gamma$ in $M-S$ which is homotopic to $C$ in $M-S$.

Consider the case where $M$ is a closed disk $D$. Suppose $\phi$ is a canonical homeomorphism on $D$ relative to a set $S$. Assume that $\phi$ fixes each point of $S$. Then it is easy to see that $\phi$ is equal to the identity map, id, on any finite-order component. We call such a component an id-component. Thus every component of $\phi$ is either a pseudo-Anosov component or an id-component. Let $\nu$ be the number of id-components of $\phi$. Let $b^{+}$(resp. $b^{-}$) be the number of points $x$ of $S$ such that $x$ is contained in a pseudo-Anosov component and $\phi$ fixes each prong (resp. no prongs) at $x$. Let $\operatorname{Fix}(\phi)$ denote the fixed point set of $\phi$. A slightly weaker form of Lemma 5 in [8] and Lemma 6 in [8] are combined into the following lemma, which will be used to prove Theorem 2: 
Lemma 1. Suppose $\phi: D \rightarrow D$ is a canonical homeomorphism relative to a set $S$ with $\phi \neq \mathrm{id}$ and $S \subset \operatorname{Fix}(\phi)$. Assume that $\phi$ has no fixed points on the interior of any pseudo-Anosov component. Then $\nu+b^{+}-b^{-} \geq 1+\alpha$, where $\alpha=0$ if $\partial D$ is contained in a pseudo-Anosov component and $\phi$ has a fixed point on $\partial D$, and $\alpha=1$ otherwise.

\section{Facts on disk embeddings}

We collect here some results obtained in [8] which will be used to prove the results of this paper. Let $D$ be a closed disk and $g: D \rightarrow D$ an orientationpreserving topological embedding with finitely many fixed points all of which are contained in Int $D$. Suppose an isotopy $g_{t}: D \rightarrow D(0 \leq t \leq \omega)$ with $g_{0}=$ id, $g_{\omega}=g$ is given.

Given a subset $S$ of $\operatorname{Fix}(g)$, define a braid $b(S, g)$, called the braid of $S$ with respect to $g$, by

$$
b(S, g)=\bigcup_{0 \leq t \leq \omega}\left(g_{t}(S) \times\{t\}\right) .
$$

In the remainder of this section, $b(S, g)$ will be denoted by $b(S)$ for simplicity. Two fixed points $x_{1}, x_{2}$ of $g$ are said to be $g$-equivalent if $b\left(\left\{x_{1}, x_{2}\right\}\right)$ is a block in $b(\operatorname{Fix}(g))$. Proposition 1 in [8] shows that the $g$-equivalence relation is an equivalence relation on $\operatorname{Fix}(g)$.

A fixed point $x$ of $g$ is said to be stable if for any neighbourhood $V$ of $x$, there is a neighbourhood $W$ of $x$ with $g^{m}(W) \subset V$ for any integer $m \geq 0$. Let $\psi: D \rightarrow D$ be the canonical homeomorphism in the isotopy class of $g$ relative to $\operatorname{Fix}(g)$. Since the isotopy between $g$ and $\psi$ keeps the set $\operatorname{Fix}(g)$ invariant, we have $\operatorname{Fix}(g) \subset \operatorname{Fix}(\psi)$.

Suppose $E$ is a $g$-equivalence class. The following facts on $E$, fixed points of $g$, and components of $\psi$ hold:

(i) $E$ is contained in a component of $\psi$.

(ii) Let $N_{E}$ denote the component of $\psi$ containing $E$. If $N_{E}$ is a pseudoAnosov component, then $E$ consists of a single point.

(iii) If $E$ has at least two points, then it contains an unstable fixed point.

(iv) Suppose $E$ consists of a single point $x_{0}$. If there is a subset $S$ of $\operatorname{Fix}(g)$ containing $x_{0}$ such that $b(S)$ and $b\left(S-\left\{x_{0}\right\}\right)$ are blocks in $b(\operatorname{Fix}(g))$, then $x_{0}$ is unstable.

(v) If $N_{E}$ is an id-component, then $E$ contains an unstable fixed point.

(vi) If $x \in \operatorname{Fix}(g)$ and $\psi$ fixes each prong at $x$, then $x$ is unstable.

(vii) Any id-component of $\psi$ has a fixed point of $g$.

The facts (i) and (ii) are immediate consequences of [8, Lemma 2]. (iii), (iv) are Theorem 3 and Proposition 3 in [8], respectively. (v)-(vii) are contained in Section 9 of [8]. 
For blocks of fixed points, the following fact hold:

Lemma 2. Suppose $S_{0}, S$ are two subsets of $\operatorname{Fix}(g)$ with $S_{0} \subset S$. Then the following conditions are equivalent:

(i) $b\left(S_{0}\right)$ is a block in $b(S)$.

(ii) There is a simple closed curve $C$ in $D-S$ such that $S_{0}$ is equal to the set of fixed points of $g$ enclosed by $C$ and that $C$ is homotopic to its image $g(C)$ in $D-S$.

(iii) There is a $\psi$-invariant curve $\Gamma$ in $D-S$ such that $S_{0}$ is equal to the set of fixed points of $g$ enclosed by $\Gamma$.

The equivalence of (i) and (ii) is Lemma 1 in [8]. The equivalence of (ii) and (iii) follows easily from the fact on invariant curves stated in Section 5.

\section{Proofs of the results}

For $0 \leq t \leq \omega$, let $U_{t}: \mathbb{R}^{2} \rightarrow \mathbb{R}^{2}$ be the translation operator defined by $U_{t}\left(x_{0}\right)=x(t)$ for $x_{0} \in \mathbb{R}^{2}$, where $x(t)$ is the solution of (1) with initial-value $x_{0}$ (see [7]). Note that a point of $\mathbb{R}^{2}$ is the initial-value of some $\omega$-periodic solution if and only if it is a fixed point of $U_{\omega}$. Therefore, since there are only finitely many $\omega$-periodic solutions, the fixed point set $\operatorname{Fix}\left(U_{\omega}\right)$ is bounded. Hence, one can choose a closed disk $D_{0}$ with $\operatorname{Fix}\left(U_{\omega}\right) \subset \operatorname{Int} D_{0}$. Also, choose a closed disk $D$ with $D_{0} \cup U_{\omega}\left(D_{0}\right) \subset \operatorname{Int} D$. Let $\Phi_{t}: D \rightarrow \mathbb{R}^{2}$ be the restriction of $U_{t}$ to the disk $D$, and let $\Phi=\Phi_{\omega}$. Then $\operatorname{Fix}(\Phi)=\operatorname{Fix}\left(U_{\omega}\right) \subset \operatorname{Int} D$. Choose a closed disk $D^{\prime}$ with $\Phi_{t}(D) \subset \operatorname{Int} D^{\prime}$ for any $t \in[0, \omega]$. Then the isotopy $\Phi_{t}: D \rightarrow D^{\prime}$ of embeddings can be extended to an isotopy $\Phi_{t}^{\prime}: D^{\prime} \rightarrow D^{\prime}$ of homeomorphisms. Let $\Phi^{\prime}=\Phi_{\omega}^{\prime}: D^{\prime} \rightarrow D^{\prime}$. Note that $\Phi^{\prime}$ is an extension of $\Phi$ to $D^{\prime}$, and so $\operatorname{Fix}(\Phi) \subset \operatorname{Fix}\left(\Phi^{\prime}\right)$. Since $\Phi$ has no fixed points on some neighbourhood of $\partial D$, we can take $\Phi_{t}^{\prime}$ so that $\Phi^{\prime}$ has finitely many fixed points on $D^{\prime}-D$ and moreover has no fixed points on $\partial D^{\prime}$. Thus $\operatorname{Fix}\left(\Phi^{\prime}\right)$ is a finite set contained in $\operatorname{Int} D^{\prime}$.

For any subset $S$ of $\operatorname{Fix}\left(\Phi^{\prime}\right)$, let $b(S)$ denote the braid $b\left(S, \Phi^{\prime}\right)$. Suppose $\mathcal{P}$ is a subset of $\mathcal{P}_{\omega}$ and let $S$ be the set of the initial-values of solutions in $\mathcal{P}$, i.e., $S=\{\xi(0) \mid \xi \in \mathcal{P}\}$. Then we have $b(S)=b(\mathcal{P})$, since $\Phi_{t}^{\prime}(\xi(0))=U_{t}(\xi(0))=\xi(t)$ for any $t, \xi \in \mathcal{P}$. In particular, we have $b(\operatorname{Fix}(\Phi))=b\left(\mathcal{P}_{\omega}\right)$.

Lemma 3. Let $S$ be a subset of $\operatorname{Fix}(\Phi)$. Then $b(S)$ is a block in $b(\operatorname{Fix}(\Phi))$ if and only if it is a block in $b\left(\operatorname{Fix}\left(\Phi^{\prime}\right)\right)$.

Proof. It is obvious that if $b(S)$ is a block in $b\left(\operatorname{Fix}\left(\Phi^{\prime}\right)\right)$, then it is a block in $b(\operatorname{Fix}(\Phi))$, since $b(S) \subset b(\operatorname{Fix}(\Phi)) \subset b\left(\operatorname{Fix}\left(\Phi^{\prime}\right)\right)$. Conversely, assume $b(S)$ is a block in $b(\operatorname{Fix}(\Phi))$. Then, replacing $S_{0}, S, g, D$ in Lemma 2 with $S, \operatorname{Fix}(\Phi), \Phi^{\prime}, D^{\prime}$ respectively, we have that there is a simple closed curve $C$ in $D^{\prime}-\operatorname{Fix}(\Phi)$ such that $S$ is equal to the set of fixed points of $\Phi^{\prime}$ enclosed by $C$ and furthermore 
$C$ is homotopic to its image $\Phi^{\prime}(C)$ in $D^{\prime}-\operatorname{Fix}(\Phi)$. Choose a homotopy $\gamma_{t}$ : $S^{1} \rightarrow D^{\prime}-\operatorname{Fix}(\Phi) \quad(0 \leq t \leq 1)$ with $\gamma_{0}\left(S^{1}\right)=C$ and $\gamma_{1}\left(S^{1}\right)=\Phi^{\prime}(C)$. We can assume, without loss of generality, that $C$ is contained in $D_{0}$, since there are no fixed points of $\Phi$ on $D^{\prime}-D_{0}$. Then $\Phi^{\prime}(C)=\Phi(C) \subset \Phi\left(D_{0}\right)=U_{\omega}\left(D_{0}\right)$. Choose a closed disk $\Delta$ with $D_{0} \cup U_{\omega}\left(D_{0}\right) \subset \Delta \subset$ Int $D$, and let $r: D^{\prime} \rightarrow D$ be a homeomorphism with $r=$ id on $\Delta$. Then $r \circ \gamma_{t}: S^{1} \rightarrow D-\operatorname{Fix}(\Phi)$ induces a homotopy between $r(C)$ and $r\left(\Phi^{\prime}(C)\right)$. Since $C \cup \Phi^{\prime}(C) \subset \Delta$, we have $r(C)=C$ and $r\left(\Phi^{\prime}(C)\right)=\Phi^{\prime}(C)$. Therefore, $C$ and $\Phi^{\prime}(C)$ are homotopic in $D-\operatorname{Fix}(\Phi)$ and hence they are homotopic in the larger set $D^{\prime}-\operatorname{Fix}\left(\Phi^{\prime}\right)$. Thus, by Lemma 2 , $b(S)$ is a block in $b\left(\operatorname{Fix}\left(\Phi^{\prime}\right)\right)$.

We say two points $x_{1}, x_{2}$ in $\operatorname{Fix}(\Phi)$ are $\Phi$-equivalent if $b\left(\left\{x_{1}, x_{2}\right\}\right)$ is a block in $b(\operatorname{Fix}(\Phi))$. Note that $\Phi^{\prime}$ satisfies the same assumption as those imposed on $g$ in Section 6. Therefore, replacing $g$ with $\Phi^{\prime}$ in the definition of the $g$-equivalence relation, we obtain the $\Phi^{\prime}$-equivalence relation: Fixed points $x_{1}, x_{2}$ of $\Phi^{\prime}$ are $\Phi^{\prime}$-equivalent if $b\left(\left\{x_{1}, x_{2}\right\}\right)$ forms a block in $b\left(\operatorname{Fix}\left(\Phi^{\prime}\right)\right)$. This is an equivalence relation on $\operatorname{Fix}\left(\Phi^{\prime}\right)$.

Lemma 4. Suppose $\xi_{1}, \xi_{2}$ are $\omega$-periodic solutions, and let $x_{1}, x_{2}$ be their initial-values. Then the following conditions are equivalent:

(i) $\xi_{1}$ and $\xi_{2}$ are equivalent.

(ii) $x_{1}$ and $x_{2}$ are $\Phi$-equivalent.

(iii) $x_{1}$ and $x_{2}$ are $\Phi^{\prime}$-equivalent.

Proof. The equivalence of the conditions (i) and (ii) follows easily from the equalities $b\left(\mathcal{P}_{\omega}\right)=b(\operatorname{Fix}(\Phi)), b\left(\left\{\xi_{1}, \xi_{2}\right\}\right)=b\left(\left\{x_{1}, x_{2}\right\}\right)$. Also, the equivalence of (ii) and (iii) follows easily from Lemma 3.

Proof of Proposition 1. Since the $\Phi^{\prime}$-equivalence relation is an equivalence relation, the equivalence of (i) and (iii) in Lemma 4 implies that the relation on $\mathcal{P}_{\omega}$ is also an equivalence relation.

A fixed point $x$ of $\Phi$ is said to be peripheral if it is the initial-value of a peripheral $\omega$-periodic solution. In other words, $x$ is peripheral if $\operatorname{Fix}(\Phi)=\{x\}$ or $b(\operatorname{Fix}(\Phi)-\{x\})$ is a block in $b(\operatorname{Fix}(\Phi))$. Lemma 4 implies that the $\Phi$-equivalence relation is an equivalence relation on $\operatorname{Fix}(\Phi)$. A $\Phi$-equivalence class is said to be non-peripheral if it contains no peripheral fixed points.

Let $\phi^{\prime}: D^{\prime} \rightarrow D^{\prime}$ be the canonical homeomorphism in the isotopy class of $\Phi^{\prime}$ relative to $\operatorname{Fix}\left(\Phi^{\prime}\right)$. Since $\phi^{\prime}$ fixes each point of $\operatorname{Fix}\left(\Phi^{\prime}\right)$, any component of $\phi^{\prime}$ is a pseudo-Anosov component or an id-component, as has been shown in Section 5 . Let $C_{0}=\partial D_{0}$. Then $C_{0}$ and $\Phi^{\prime}\left(C_{0}\right)$ are simple closed curves in $D$ enclosing $\operatorname{Fix}(\Phi)$, and hence they are homotopic to the boundary circle $\partial D$ of $D$ in the punctured disk $D^{\prime}-\operatorname{Fix}\left(\Phi^{\prime}\right)$. Therefore they are homotopic in $D^{\prime}-\operatorname{Fix}\left(\Phi^{\prime}\right)$ 
mutually. As has been noted in Section 5, this implies that there exists a $\phi^{\prime}$ invariant curve $\Gamma$ contained in $D^{\prime}-\operatorname{Fix}\left(\Phi^{\prime}\right)$ such that $\operatorname{Fix}(\Phi)$ coincides with the set of fixed points of $\Phi^{\prime}$ enclosed by $\Gamma$. Let $M$ be the closure of the interior domain of $\Gamma$. Then $\operatorname{Fix}\left(\Phi^{\prime}\right) \cap M=\operatorname{Fix}(\Phi)$.

Suppose $x_{0} \in \operatorname{Fix}(\Phi)$ and let $N$ be the component of $\phi^{\prime}$ containing $x_{0}$.

Lemma 5. A necessary and sufficient condition for $x_{0}$ to be peripheral is that $N$ contains $\Gamma$ and is an id-component of $\phi^{\prime}$.

Proof. Assume that $x_{0}$ is peripheral. We claim that there exists a $\phi^{\prime}$ invariant set $M_{0}$ with $\operatorname{Fix}\left(\Phi^{\prime}\right) \cap M_{0}=\left\{x_{0}\right\}$ and $M_{0} \cap \Gamma \neq \emptyset$. In the case of $\operatorname{Fix}(\Phi)=\left\{x_{0}\right\}$, let $M_{0}=M$. Then it has the required property. Consider next the case where $\operatorname{Fix}(\Phi) \neq\left\{x_{0}\right\}$. Since $x_{0}$ is peripheral, $b\left(\operatorname{Fix}(\Phi)-\left\{x_{0}\right\}\right)$ is a block in $b(\operatorname{Fix}(\Phi))$ and hence in $b\left(\operatorname{Fix}\left(\Phi^{\prime}\right)\right)$ by Lemma 3 . Therefore, by Lemma 2 , there is a $\phi^{\prime}$-invariant curve $\bar{\Gamma}$ in $D^{\prime}-\operatorname{Fix}\left(\Phi^{\prime}\right)$ such that the set of fixed points of $\Phi^{\prime}$ enclosed by $\bar{\Gamma}$ is equal to $\operatorname{Fix}(\Phi)-\left\{x_{0}\right\}$. Let $L$ be the closure of the outer connected component of $D^{\prime}-\bar{\Gamma}$. Let $M_{0}$ be the connected component of $M \cap L$ containing $x_{0}$. Then it is easy to see that $M_{0}$ has the required property.

Since $M_{0}$ is a $\phi^{\prime}$-invariant set containing only one fixed point, $x_{0}$, of $\Phi^{\prime}$, the canonical homeomorphism $\phi^{\prime}$ relative to $\operatorname{Fix}\left(\Phi^{\prime}\right)$ must be the identity on $M_{0}$. This implies that $N$ contains $M_{0}$, and it is an id-component. Also, since $M_{0}$ intersects with $\Gamma$, we have $\Gamma \subset N$. The converse assertion is easily proved.

Replacing $g, g_{t}, \psi$ in Section 6 with $\Phi^{\prime}, \Phi_{t}^{\prime}, \phi^{\prime}$, we can apply the facts there to $\Phi^{\prime}$-equivalence classes and components of $\phi^{\prime}$. In particular, applying (i) in Section 6 to $\Phi^{\prime}$, we see that each $\Phi^{\prime}$-equivalence class $E^{\prime}$ is contained in a component of $\phi^{\prime}$ denoted by $N_{E^{\prime}}$.

Lemma 6. Let E be a non-peripheral $\Phi$-equivalence class. Then

(i) $E$ is a $\Phi^{\prime}$-equivalence class, and

(ii) the component $N_{E}$ of $\phi^{\prime}$ is contained in $M$.

Proof. By Lemma 4, we see that $E$ is contained in a $\Phi^{\prime}$-equivalence class $E^{\prime}$. We claim that $\Gamma$ does not intersect $\operatorname{Int} N_{E^{\prime}}$. Consider the case where $N_{E^{\prime}}$ is a pseudo-Anosov component. Then, since $\Gamma$ is a $\phi^{\prime}$-invariant curve, it cannot intersect Int $N_{E^{\prime}}$. Consider the case where $N_{E^{\prime}}$ is an id-component. If it contains $\Gamma$, then by Lemma $5, E$ must have a peripheral point. This contradicts with $E$ being non-peripheral, Thus it is proved that $N_{E^{\prime}} \cap \Gamma=\emptyset$, and the claim is proved. The claim implies that $N_{E^{\prime}} \subset M$. In particular, we have $E^{\prime} \subset M$, and hence $E^{\prime} \cap M=E^{\prime}$. Since $E^{\prime} \cap M$ is equal to $E$, this implies that $E=E^{\prime}$, which is a $\Phi^{\prime}$-equivalence class, and $N_{E}=N_{E^{\prime}} \subset M$. Thus the lemma is proved.

Proof of Proposition 2. Let $\xi_{1}, \xi_{2}$ be peripheral $\omega$-periodic solutions, and let $x_{1}, x_{2}$ be their initial-values. Let $N_{\Gamma}$ be the component of $\phi^{\prime}$ containing $\Gamma$. 
By Lemma $5, x_{1}$ and $x_{2}$ are contained in $N_{\Gamma}$, and $N_{\Gamma}$ is an id-component. These imply that there is a $\phi^{\prime}$-invariant curve in $D^{\prime}-\operatorname{Fix}\left(\Phi^{\prime}\right)$ such that $x_{1}, x_{2}$ are the only fixed points of $\Phi^{\prime}$ enclosed by it. Hence by Lemma $2, b\left(\left\{x_{1}, x_{2}\right\}\right)$ is a block in $b\left(\operatorname{Fix}\left(\Phi^{\prime}\right)\right)$, and therefore $x_{1}$ and $x_{2}$ are $\Phi^{\prime}$-equivalent. By Lemma 4 , we have that $\xi_{1}, \xi_{2}$ are equivalent.

Proof of TheOREM 1. Let $\mathcal{E}$ be a non-peripheral equivalence class consisting of at least two $\omega$-periodic solutions. Then, the set $E$ of initial-values of the solutions in $\mathcal{E}$ is a non-peripheral $\Phi$-equivalence class, and hence by Lemma 6 , it is a $\Phi^{\prime}$-equivalence class. Therefore, since $E$ has at least two points, by (iii) in Section 6 , we have that $E$ contains an unstable fixed point of $\Phi^{\prime}$, which is also an unstable fixed point of $U_{\omega}$, since $\Phi^{\prime}=U_{\omega}$ on $D$. Since an $\omega$-periodic solution is unstable if and only if its initial-value is an unstable fixed point of $U_{\omega}$ (see Lemma 9.1 in [7]), we conclude that $\mathcal{E}$ has an unstable solution.

Proof of Proposition 3. Let $x_{0}=\xi_{0}(0), E=\left\{x_{0}\right\}$, and $S$ the set of initial-values of the solutions in $\mathcal{P}$. Then $E$ is a non-peripheral $\Phi$-equivalence class and hence it is a $\Phi^{\prime}$-equivalence class. Moreover, since $b(S)=b(\mathcal{P}), b(S-$ $\left.\left\{x_{0}\right\}\right)=b\left(\mathcal{P}-\left\{\xi_{0}\right\}\right)$, we have that $b(S), b\left(S-\left\{x_{0}\right\}\right)$ are blocks in $b(\operatorname{Fix}(\Phi))$. Hence they are blocks in $b\left(\operatorname{Fix}\left(\Phi^{\prime}\right)\right)$ by Lemma 3 . Therefore, by (iv) in Section 6 , we see that $x_{0}$ is unstable, and consequently so is $\xi_{0}$.

Proof of Theorem 2. Let $c$ be the number of non-peripheral $\Phi$-equivalence classes, and $c_{u}$ the number of such equivalence classes containing an unstable fixed point. For the proof, it is enough to see that $2 c_{u}>c$.

Let $\mathcal{C}$ be the set of non-peripheral $\Phi$-equivalence classes. By Lemma 6 , every member of $\mathcal{C}$ is a $\Phi^{\prime}$-equivalence class. Therefore, the fact (ii) in Section 6 implies that $\mathcal{C}$ is decomposed into a disjoint union $\mathcal{C}=\mathcal{C}_{0} \cup \mathcal{C}_{+} \cup \mathcal{C}_{-}$, where $E \in \mathcal{C}$ belongs to $\mathcal{C}_{0}$ if $N_{E}$ is an id-component, and $E$ belongs to $\mathcal{C}_{+}\left(\right.$resp. $\mathcal{C}_{-}$) if $N_{E}$ is a pseudo-Anosov component and $\phi^{\prime}$ fixes each prong (resp. no prongs) at the unique point in $E$. By (v) and (vi) in Section 6, any $\Phi$-equivalence class in $\mathcal{C}_{0} \cup \mathcal{C}_{+}$contains an unstable fixed point. Hence if we let $c_{e}=\sharp \mathcal{C}_{e}$, the cardinality of $\mathcal{C}_{e}$, for $e=0,+,-$, then we have $c_{u} \geq c_{0}+c_{+}$. Since $c=c_{0}+c_{+}+c_{-}$, this implies that

$$
2 c_{u}-c \geq 2\left(c_{0}+c_{+}\right)-\left(c_{0}+c_{+}+c_{-}\right)=c_{0}+c_{+}-c_{-} .
$$

Therefore, for the proof of the desired inequality $2 c_{u}>c$, it is enough to see that

$$
c_{0}+c_{+}-c_{-}>0 \text {. }
$$

Let $\phi$ be the restriction of $\phi^{\prime}: D^{\prime} \rightarrow D^{\prime}$ to $M$. We can assume without loss of generality that this map $\phi$ is a canonical homeomorphism on $M$ relative to $\operatorname{Fix}(\Phi)$. Since $M$ is a disk and $\phi$ fixes each point of $\operatorname{Fix}(\Phi)$, we can define the integres $\nu, b^{+}, b^{-}$as in Section 5 . 
Let Fix $_{+}(\Phi)($ resp. Fix $-(\Phi))$ be the set of points $x$ of $\operatorname{Fix}(\Phi)$ such that $x$ is contained in a pseudo-Anosov component of $\phi^{\prime}$ and $\phi^{\prime}$ has an invariant prong (resp. no invariant prongs) at $x$. Let $e=+,-$. Then $b^{e}=\sharp \operatorname{Fix}_{e}(\Phi)$. Let $\rho_{e}: \mathcal{C}_{e} \rightarrow \operatorname{Fix}_{e}(\Phi)$ be the injective map which sends $E \in \mathcal{C}_{e}$ to the unique point in $E$. Suppose $x_{0} \in \operatorname{Fix}_{e}(\Phi)$. Let $E=\left\{x_{0}\right\}$. If $x_{0}$ is $\Phi^{\prime}$-equivalent to another fixed point of $\Phi^{\prime}$, then by (ii) in Section $6, x_{0}$ is contained in an id-component of $\phi^{\prime}$, and we get a contradiction. Hence $E$ is a $\Phi^{\prime}$-equivalence class. Therefore, Lemma 4 implies that $E$ is a $\Phi$-equivalence class, and by Lemma $5, E$ is nonperipheral. Hence $E \in \mathcal{C}_{e}$ and $\rho_{e}(E)=x_{0}$, and so the map $\rho_{e}$ is onto. Therefore we have

$$
c_{+}=b^{+}, \quad c_{-}=b^{-} .
$$

Let $I(\phi)$ be the set of id-components of $\phi$ with no intersection with $\Gamma$. Suppose $E \in \mathcal{C}_{0}$. By Lemma $5, N_{E}$ has no intersection with $\Gamma$, and so $N_{E} \subset M$. Hence $N_{E}$ is a component of $\phi$, and so we have $N_{E} \in I(\phi)$. Therefore we can define an injective map $\rho_{0}: \mathcal{C}_{0} \rightarrow I(\phi)$ which sends $E \in \mathcal{C}_{0}$ to $N_{E}$.

Suppose $N \in I(\phi)$. Then since $N$ has no intersection with $\Gamma$, it is a component of $\phi^{\prime}$. Moreover, it is an id-component and hence the fact (vii) in Section 6 implies that it contains a fixed point of $\Phi^{\prime}$. Choose such a point $x_{0}$. Since $N$ is contained in $M, x_{0}$ is a fixed point of $\Phi$. Let $E$ be the $\Phi$-equivalence class containing $x_{0}$. Then since $N \cap \Gamma=\emptyset$, by Lemma $5, x_{0}$ is non-peripheral. Therefore $E$ is a non-peripheral $\Phi$-equivalence class. Since $N_{E} \subset M$, by Lemma 6(ii), and both $N_{E}$ and $N$ contain $x_{0}$, they coincide. Hence $N_{E}$ is an id-component. Therefore $E \in \mathcal{C}_{0}$ and $\rho_{0}(E)=N_{E}=N$. Therefore $\rho_{0}$ is onto, and hence $c_{0}=\sharp \mathcal{C}_{0}=\sharp I(\phi)$.

Consider the case where there is no peripheral fixed point of $\Phi$. Then, by Lemma 5, any id-component of $\phi^{\prime}$ has no intersection with $\Gamma$. Thus clearly we have $\sharp I(\phi)=\nu$ and hence $c_{0}=\nu$. Hence, by (3) and Lemma 1 , the inequality (2) holds.

Consider the case where there is a peripheral fixed point of $\Phi$. Then, by Lemma $5, \Gamma$ is contained in an id-component, and hence $c_{0}=\sharp I(\phi)=\nu-1$ and $\alpha=1$. Therefore (2) holds by (3) and Lemma 1. Thus the proof is completed. $\square$

\section{REFERENCES}

[1] J. ANDREs, Existence, uniqueness, and instability of large-period harmonics to the thirdorder nonlinear ordinary differential equations, J. Math. Anal. Appl. 199 (1996), 445457.

[2] Concluding remarks to problem of Moser and conjecture of Mawhin, Ann. Math. Sil. 10 (1996), 57-65. 
[3] J. S. Birman, Braids, Links, and Mapping Class Groups, Ann. Math. Studies, vol. 82, Princeton Univ. Press, Princeton, 1974.

[4] P. Boyland, Topological methods in surface dynamics, Topology Appl. 58 (1994), 223298.

[5] A. Fathi, F. Laudenbach and V. Poénaru, Travaux de Thurston sur les surfaces, Astérisque 66-67 (1979).

[6] V. L. Hansen, Braids and Coverings: Selected Topics, London Math. Soc. Student Texts, vol. 18, Cambridge Univ. Press, Cambridge, 1989.

[7] M. A. Krasnosel'skil, The Operator of Translation Along the Trajectories of Differential Equations, Translations of Math. Monographs,, vol. 19, Amer. Math. Soc., 1968.

[8] T. MATSuOKA, Fixed point index and braid invariant for fixed points of embeddings on the disk, preprint.

[9] F. A. McRobie And J. M. T. Thompson, Braids and knots in driven oscillators, Internat. J. Bifur. Chaos appl. Sci. Engrg. 3 (1993), 1343-1361.

[10] W. P. Thurston, On the geometry and dynamics of diffeomorphisms of surfaces, Bull. Amer. Math. Soc. 19 (1988), 417-431.

Manuscript received December 1, 1999

TAKASHi MATSUOKA

Department of Mathematics

Naruto University of Education

Takashima, Naruto 772-8502, JAPAN

E-mail address: matsuoka@naruto-u.ac.jp 